\title{
Diane Lamoureux
}

professeure, département de science politique, Université Laval

(1991)

\section{"Le rôle des intellectuelles et intellectuels en sciences sociales dans le débat politique actuel"}

Un document produit en version numérique par Jean-Marie Tremblay, bénévole, professeur de sociologie au Cégep de Chicoutimi

Courriel: jmt sociologue@,videotron.ca

Site web pédagogique : http://www.uqac.ca/jmt-sociologue/

Dans le cadre de la collection: "Les classiques des sciences sociales"

Site web: http://www.uqac.ca/Classiques_des_sciences_sociales

Une collection développée en collaboration avec la Bibliothèque Paul-Émile-Boulet de l'Université du Québec à Chicoutimi Site web: http://bibliotheque.uqac.ca/ 
Cette édition électronique a été réalisée par Jean-Marie Tremblay, bénévole, professeur de sociologie au Cégep de Chicoutimi à partir de :

Diane Lamoureux, "Le rôle des intellectuelles et intellectuels en sciences sociales dans le débat politique actuel”.

Un article publié dans l'ouvrage sous la direction de Réjean Pelletier, Guy Laforest et Vincent Lemieux, Le Québec et la restructuration du Canada, 1980-1992: enjeux et perspectives, chapitre 10, pp. 199-215. Québec : Éditions du Septentrion, 1991, 312 pp.

Mme Lamoureux est professeure au département de science politique à l’Université Laval.

[Autorisation accordée par l'auteure le 2 février 2004 et reconfirmée le 28 mars 2004]

g- Courriel : Diane.Lamoureux@pol.ulaval.ca

Polices de caractères utilisée :

Pour le texte: Times, 12 points.

Pour les citations : Times 10 points.

Pour les notes de bas de page : Times, 10 points.

Édition électronique réalisée avec le traitement de textes Microsoft Word 2001 pour Macintosh.

Mise en page sur papier format : LETTRE (US letter), $8.5^{\prime \prime} \times 11$ '')

Édition numérique réalisée le 20 juillet 2005 à Chicoutimi, Ville de Saguenay, province de Québec, Canada.

\section{Fait avec}




\section{Table des matières}

Présentation du texte et de l'auteur

Introduction

Un rôle marginal

Les tentations de l'expertise

Les pièges de l'idéologie

Des voies d'intervention 


\section{Diane Lamoureux}

[politologue, Université Laval]

\section{"Le rôle des intellectuelles et intellectuels en sciences sociales dans le débat politique actuel".}

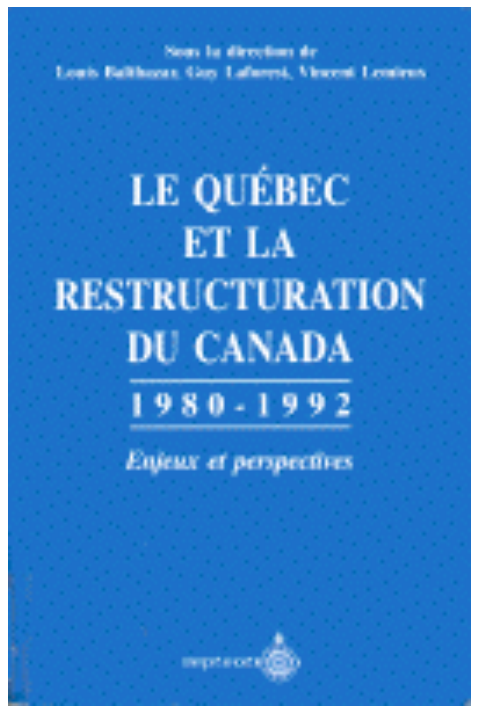

Un article publié dans l'ouvrage sous la direction de Réjean Pelletier, Guy Laforest et Vincent Lemieux, Le Québec et la restructuration du Canada, 1980-1992: enjeux et perspectives, chapitre 10, pp. 199-215. Québec : Éditions du Septentrion, 1991, 312 pp. 


\title{
Présentation du texte
}

\author{
Diane Lamoureux
}

$\underline{\text { Retour à la table des matières }}$

Les intellectuels sont loin d'être toujours d'accord entre eux. Les textes écrits dans ce livre par Guy Laforest et Max Nemni le démontrent bien. Quel devrait être leur rôle dans la crise que nous traversons? Dans sa réponse à cette interrogation, Diane Lamoureux commence par noter le rôle mineur joué par les intellectuels dans les grands débats de l'heure. Cela tient d'une part à des changements au sein des disciplines que sont les sciences sociales et humaines. Dans les années quatre-vingt, les intellectuels ont évacué l'espace public pour se réfugier dans un certain silence. Plusieurs ont succombé à l'appel de l'expertise. Cette dimension technique a prévalu à la Commission Bélanger-Campeau. D'autres se sont tus après avoir abdiqué leur sens critique. À ce propos, l'auteure réfléchit aux effets politiques de la vogue idéologique marxiste au Québec. En définitive, elle croit que revient aux intellectuels le rôle de susciter un débat public pluraliste, où personne n'aura le monopole de la vérité. Diane Lamoureux est professeure agrégée au département de science politique de l'Université Laval. 
Diane Lamoureux

"Le rôle des intellectuelles et intellectuels en sciences sociales dans le débat politique actuel".

\section{Introduction}

Dire le vrai, telle est la seule responsabilité des intellectuels en tant qu'intellectuels. Sortis de cette voie, ils sont des citoyens, ils sont en politique et défendent leur opinion. C'est leur droit. Mais nous n'avons pas le droit de proclamer que parce que nous sommes des intellectuels nous sommes la conscience de la nation ${ }^{1}$.

Historiquement, les sciences sociales ont joué un rôle important dans les questionnements politiques qui ont agité la société québécoise. On n'a qu'à se rappeler l'impact d'un Lionel Groulx ou d'un André Laurendeau dans la formation du nationalisme canadien-français, le rôle de la Faculté des sciences sociales de l'Université Laval dans l'opposition au régime Duplessis, le rôle des diplômées et diplômés en sciences sociales dans les comités de citoyens, dans la modernisation de l'État québécois, dans le renouvellement du syndicalisme -principalement dans le secteur publie à la fin des années 60 et au début des années 70 - dans la mise

1 Hannah Arendt, «Les intellectuels et la responsabilité », Cahiers du GRIF, 33, 1986 (nouvelle édition augmentée, 1991), p. 151. 
sur pied du Parti québécois et dans la résurgence du nationalisme, pour ne citer que quelques exemples.

Cette implication a pris des formes extrêmement diversifiées qu'on peut cependant regrouper autour des axes suivants. D'abord, la consolidation des universités elles-mêmes et plus particulièrement de leur secteur sciences sociales, ce qui permettait d'assurer la relève en plus de fournir des emplois. Ensuite, des revues, usuellement fortement homogènes d'un point de vue idéologique, qui servent de relais entre l'élaboration théorique et le public en général en plus de permettre de cristalliser les opinion ${ }^{2}$. Finalement, une présence soutenue sur la scène publique, ce qui peut prendre la forme d'une collaboration régulière aux médias ${ }^{3}$, principalement écrits, ou des prises de parole sur les grands sujets de l'actualité politique.

Tout ceci implique que c'est, dans une large mesure, à partir d'une extension de leur pratique professionnelle que les intellectuelles et intellectuels choisissent d'intervenir dans le débat public. Or une première question à se poser, c'est la base à partir de laquelle nous intervenons. Je ne pense pas qu'il soit possible d'échapper complètement à la responsabilité sociale des intellectuelles et intellectuels; notre métier en sciences sociales est de réfléchir sur le social, il est donc normal que nous ayons des opinions sur le sujet. Même celui qui a le plus contribué à opérer la distinction entre jugement de fait et jugement de valeur, Max Weber, ouvre la possibilité à une intervention citoyenne des universitaires, tout en précisant le contexte dans laquelle elle doit se dérouler :

Le professeur qui se sent la vocation de conseiller la jeunesse et qui jouit de sa confiance doit s'acquitter de ce rôle dans le contact personnel d'homme à homme. S'il se sent appelé à participer aux luttes entre les conceptions du monde et les opinions des partis, il lui est loisible de le faire hors de la salle de cours, sur la place publique, c'est-à-dire dans la presse, dans les réunions publiques, dans les associations, bref partout où il le voudra. Il est en effet par trop commode de montrer son courage de partisan en un endroit où les assistants, et peut-être les opposants, sont condamnés au silence ${ }^{4}$.

On ne saurait certes confondre l'université allemande du début du siècle avec l'université québécoise contemporaine, mais Weber souligne un phénomène qui est encore d'actualité : l'intervention politique ne peut se faire que dans un contexte où le débat est possible. Pour exercer leur jugement sur les affaires

2 Voir à ce sujet l'article d'Andrée Fortin « Les intellectuels à travers leurs revues », Recherches sociographiques, XXXI, 2, 1990.

3 On n'a qu'à penser à l'activité journalistique d'André Laurendeau, Gérard Pelletier ou Gérard Bergeron pour ne citer que quelques exemples relativement anciens.

4 Max Weber, Le savant et le politique, Paris, UGE, Collection 10/18, 1979, p. 88. [Texte disponible dans Les Classiques des sciences sociales. JMT.] 
publiques, les intellectuelles et intellectuels ont d'abord et avant tout besoin d'un espace public reposant sur la possibilité de confrontation entre les points de vue et d'examen critique des options. En partant de ces constats, j'aimerais explorer les pistes suivantes : le rôle relativement congru des intellectuelles et intellectuels dans les débats politiques actuels; le rapport entre ce phénomène et les transformations qui se sont manifestées dans le champ des sciences sociales au cours des dernières décennies; l'oscillation entre un rôle idéologique et un rôle d'expertise. Tout ceci servira à analyser la marge de manœuvre dont nous pouvons disposer si nous estimons qu'il nous revient de maintenir et de participer aux débats sociaux.

Ce type de raisonnement rejoint en partie les voies d'avenir pour la Faculté des sciences sociales de l'Université Laval que traçait Léon Dion lors de son allocution à l'occasion du colloque marquant le 50e anniversaire de cette Faculté. Celui-ci insistait d'une part sur la nécessité de mettre fin à l'hyperspécialisation qui caractérise les sciences sociales

... la Faculté doit se réorienter : elle doit d'abord reprendre la réforme de ses programmes dans deux directions : d'une part, alléger son baccalauréat disciplinaire départemental (...) et d'autre part, être le pivot d'un baccalauréat en sciences humaines qui offrirait le type de formation fondamentale que réclamait le Rapport de la Commission d'étude sur l'avenir de l'Université Laval en 1979 et que réclament avec instance les dirigeants sociaux ${ }^{5}$.

D'autre part, il préconisait un retour aux sources des membres de la Faculté, estimant que l'une de ses richesses avait été sa capacité de soutien aux débats sociaux. Léon Dion esquissait ainsi ce qu'il estimait être le rôle social des intellectuelles et intellectuels «qui est, en même temps que de contribuer à la compréhension du présent, d'entrouvrir les voies de l'avenir ${ }^{6} »$.

Ses membres ensuite, chacun en assumant la responsabilité de sa liberté, doivent raviver leur esprit critique et faire de la Faculté un lieu privilégié, non seulement d'analyse positive et de service, mais aussi d'animation, de discussion, de débats publics, voire de contestation des idées et des conduites reçues qui apparaîtraient anachroniques ou néfastes, de même que de production d'idées nouvelles ${ }^{7}$.

Pour cerner ce dont je veux parler, je partirai d'une définition assez générale $\mathrm{du}$ terme «intellectuel» pour y faire entrer toutes les personnes qui font

5 Léon Dion, «Nos institutions : considérations liminaires », in Les institutions québécoises : leur rôle, leur avenir, Québec, Presses de l'Université Laval, 1990, p. 47. [Texte bientôt disponible dans Les Classiques des sciences sociales. JMT.]

6 Loc. cit.

7 Loc. cit. 
profession de penser et qui se préoccupent de la vie de l'esprit ${ }^{8}$. Je suis cependant consciente de l'ambivalence du terme, je préfère m'en remettre aux définitions des dictionnaires les plus usuels pour éviter un long développement sur la notion même.

\section{Un rôle marginal}

\section{$\underline{\text { Retour à la table des matières }}$}

Au cours des années 80, le discours dominant a été celui du silence des intellectuels. Après avoir été fort présents dans les débats publics des années 60 et 70 et prêté leur voix et leur aval à diverses options politiques, les intellectuels, principalement dans le domaine des sciences sociales, profitant de leurs positions dans les institutions universitaires, ont semblé se replier dans leur tour d'ivoire. Cette appréciation a été largement médiatisée avec le film de Denys Arcand, Le déclin de l'empire américain, mais elle a également fait l'objet d'un traitement de la part des intellectuels eux-mêmes ${ }^{9}$.

Cependant, depuis l'échec de l'accord du Lac Meech, les intellectuels semblent avoir opéré un retour en force dans le champ politique. Les médias sont à l'affût de toute déclaration politique du moindre universitaire et plusieurs d'entre nous ont été appelés à plancher sur les modalités de sortie de crise. Le vœu de Léon Dion de la reprise d'une implication dans la définition de l'avenir collectif de la part des universitaires en sciences sociales semble largement exaucé : dans les tentatives de sauvetage de Meech, les intellectuels canadiens-anglais ne se sont-ils

8 À partir de maintenant j'emploierai ce terme à peu près exclusivement au masculin. Ceci n'a pas pour fonction d'alléger le texte, prétexte usuel à l'invisibilisation des femmes dans la langue française, mais plutôt de souligner la prépondérance masculine dans cette confrérie. Par ailleurs, ce groupe social, à l'instar de tous les autres groupes sociaux, est traversé par la question du genre, et être une intellectuelle n'a pas exactement la même signification qu'être un intellectuel. Pour s'en convaincre, la définition du terme par le Petit Robert est révélatrice : au masculin il s'agit d'une activité inscrite dans la division sociale du travail, au féminin, cela devient un terme péjoratif.

9 Voir à ce sujet les articles consacrés par la revue Conjoncture au silence des intellectuels, silence que cette revue tançait vertement, de même que les numéros consacrés à cette question par la nouvelle barre du jour et Possibles. À la même époque, il y a eu l'amorce d'un débat avec la publication du livre de Marc Henry Soulet, Le silence des intellectuels, Montréal, Éditions Saint-Martin, 1987. 
pas largement mobilisés et depuis, le souffle nationaliste ne continue-t-il pas de bruisser chez les spécialistes québécois des sciences sociales?

À cet égard, le colloque sur René Lévesque, qui s'est tenu en mars 1991, est particulièrement révélateur. Aux côtés de politiciens, se retrouvaient des universitaires, dont la plupart soulignaient l'actualité et la pertinence du projet souverainiste. Cependant, cette déclaration d'intention visait plus à clore le débat qu'à l'entrouvrir ; la souveraineté y apparaissait comme une fin en soi plutôt que comme un moyen d'entamer la discussion sur les modalités de la vie politique québécoise. Pourtant, l'occasion était bonne pour effectuer un retour critique sur la révolution tranquille et ses suites et poser les nouvelles questions auxquelles est confrontée la société québécoise.

De même la commission Bélanger-Campeau a fourni à plusieurs d'entre nous l'occasion d'identifier des questions politiques qu'il importe de résoudre. Il est à regretter que cela ait si peu transparu dans le rapport final des commissaires : les grandes questions politiques y ont été réduites à des aspects techniques faisant appel à des avis plus fouillés d'experts au lieu de susciter un débat collectif sur les priorités à se donner comme société. Or les «expertises» soumises à cette commission relevaient plus de l'implication citoyenne que de la science proprement dite, ce qui est parfaitement normal dans les circonstances. Ce qui l'est moins, c'est la volonté du pouvoir d'enfermer les intellectuels dans le cercle vicieux de l'expertise ou de l'idéologie.

La question de l'avenir du Québec est trop importante pour être laissée dans les mains de quelques politiciens assistés d'intervenants socio-économiques ${ }^{10}$ et aidés par quelques intellectuels qu'ils auront décidé de s'adjoindre. Prendre nos responsabilités dans les circonstances, c'est ouvrir le débat public et faire en sorte que les opinions circulent et surtout puissent se formuler. Peut-être le résultat final nous décevra-t-il, comme plusieurs d'entre nous ont été déçus en mai 80, mais au moins le débat aura-t-il été fait et nous aurons alors pleinement joué notre rôle : faire surgir un sens critique et amener nos concitoyennes et concitoyens à s'interroger sur l'avenir. Dans son plaidoyer devant ses juges, Socrate se comparait à un taon chargé d'aiguillonner la cité en y instillant le doute et « d'examiner ceux qui s'imaginent être sages et ne le sont pas ${ }^{11} »$. Notre rôle n'est donc pas de monopoliser la parole, mais plutôt de la soumettre à l'examen public.

10 Ce qui correspond à la composition de la Commission, celle-ci réunissant des élues et élus des paliers municipal, provincial et fédéral en plus des représentants des milieux des affaires et du milieu syndical.

11 Platon, Apologie de Socrate, Paris, Garnier-Flammarion, 1967, 33c, p. 45. 
C'est ce que rappelait d'ailleurs avec pertinence Habermas dans le contexte de l'unification allemande qu'on peut aisément comparer, en termes d'enjeux soulevés pour le vivre ensemble d'une communauté politique, à la conjoncture politique québécoise et canadienne. S'opposant à une unification rapide sous l'égide du roi Deutschmark, celui-ci rappelait fort à propos que

Une identité nationale qui ne s'appuie pas en premier lieu sur une compréhension de soi républicaine, procédant d'un patriotisme constitutionnel, est en opposition avec les règles universalistes requises pour que des formes de vie coexistant à égalité de droits puissent cohabiter en bonne intelligence ${ }^{12}$.

Avant de dégager quelques pistes de réflexion pour l'avenir, je voudrais commencer par disséquer le silence des années 80 qui tranche effectivement avec l'implication très forte des intellectuels en politique depuis les années 50. Au lieu de le déplorer, il me semble plus utile d'essayer de dégager des explications sur ce qui l'a rendu possible et de m'interroger sur le type de parole que devraient aujourd'hui privilégier les intellectuels.

\section{Les tentations de l'expertise}

\section{Retour à la table des matières}

Deux pistes - d'ailleurs assez complémentaires - peuvent être prises si l'on veut comprendre ce silence. D'une part, les difficultés qu'a connues l'institution universitaire au cours de cette période. De l'autre, l'échec des projets dans lesquels les intellectuels québécois avaient fortement investi - quand ils ne les ont pas euxmêmes suscités - dans les décennies précédentes : le projet indépendantiste qui connaît un recul majeur avec la défaite du référendum en mai 1980, et l'expansion du mouvement syndical et de l'extrême-gauche qui entrent tous deux en crise au début des années 80, avec les coupures dans le secteur public en 1982 et la désarticulation de la gauche m.l. dans le même temps; les prophètes d'hier ont cédé la place aux repentis honteux. Seules les intellectuelles, du fait de la montée féministe jusqu'au milieu des années 80 , ont pu échapper partiellement à cette configuration dont il importe de saisir le sens si l'on veut éviter la répétition des mêmes schémas de comportement ${ }^{13}$.

\footnotetext{
Jürgen Habermas, Écrits politiques, Paris, Cerf, 1990, p. 258.

13 Encore que là aussi l'oscillation entre l'expertise et l'idéologie ait été forte.
} 
Il importe d'emblée de souligner que la fonction intellectuelle s'est, en la quasi-absence de tout mouvement social, de plus en plus réfugiée dans les universités au cours de cette période. C'est pourquoi la crise universitaire a eu tant de répercussions sur les conditions de l'activité intellectuelle ${ }^{14}$. On doit donc chercher dans les mutations au sein des universités certains éléments explicatifs de la soudaine extinction de voix du monde intellectuel.

La crise des universités a pris plusieurs formes qui ont toutes affecté le travail des intellectuels. L'aspect le plus évident -quoique, a posteriori, il n'apparaisse pas comme ayant été le plus pernicieux - en est certainement la baisse du financement. L'éducation faisait bonne figure dans les dépenses de la révolution tranquille et les budgets qui y étaient consacrés ont généralement été à la hausse. Les brusques coupures de 1982 ont eu plusieurs conséquences: arrêt du renouvellement du corps professoral, augmentation de la charge de travail et/ou recours massif à une main-d'œuvre n'ayant pas la possibilité de faire carrière à l'université (les chargées et chargés de cours), coupures dans les services auxiliaires à l'enseignement et à la recherche (bibliothèques, perfectionnement, etc.). L'université se trouvait donc en situation de devoir justifier son existence et son rôle social.

Cela a entraîné des conséquences tant pour les profs que pour la formation universitaire. Pour les profs, l'effet le plus évident a été le repli sur soi professionnel. L'insistance a été mise sur la compétence professionnelle du corps enseignant, compétence attestée par la diplômation et la possibilité de recevoir des subventions de recherche. Le résultat a été fort probant en sciences sociales puisque le rapport sectoriel du Conseil des universités soulignait que « [le] corps professoral est fortement qualifié. Sa participation à la recherche subventionnée est élevée, comme sa production d'articles et d'ouvrages scientifiques ${ }^{15}$ ».

Ce repli sur soi professionnel allait cependant avoir pour conséquence que la vie intellectuelle s'exerce relativement peu en dehors des universités. Dans leur souci de valoriser l'institution universitaire pour éventuellement améliorer leur situation, les intellectuels de sciences sociales ont un peu perdu de vue le social, l'excellence de l'université devenant sa seule justification. De même, la remise en cause du rôle modernisateur de l'État et le morcellement des enjeux sociaux

14 Même le quotidien qui se réclamait de certaines prétentions intellectuelles, Le Devoir, a opéré un changement de cap durant ces années et Piotte a parfaitement raison de le souligner lorsqu'il mentionne la mise hors champ des intellectuels dans ce journal (cf. «L'apahasie des intellectuels » in Les avenues de la science politique. Théories, paradigmes et scientificité, Cahiers de l'ACFAS no 73, 1990, p. 230).

15 Étude sectorielle en sciences sociales, rapport final, Conseil des universités, document 23100134, 1989, p. 9. 
allaient rendre plus complexes les conditions de visibilité sociale des intellectuels dans les deux modes qu'ils ont jusqu'ici privilégiés, l'idéologie et l'expertise.

Dans le même temps, ce succès professionnel allait être associé à une perte d'attraction des sciences sociales sur les étudiantes et étudiants universitaires. Si, dans les années 60 et 70, des études en sciences sociales pouvaient donner l'impression de participer à la construction de l'avenir, cette fonction a émigré vers d'autres domaines universitaires. Le discours dominant n'est plus celui du social, mais celui de la gestion. Ceci a des effets, tant sur le recrutement étudiant ${ }^{16}$ que la formation universitaire.

La formation a été le domaine négligé des sciences sociales au cours des dernières années. La massification de la fréquentation universitaire et l'importance accrue accordée à la recherche n'ont pas été sans répercussion sur la place accordée à l'enseignement. Conséquence la plus évidente, les programmes se sont morcelés et spécialisés, au gré des recherches plutôt que selon une logique propre à l'enseignement. La généralisation de la formation à la carte ${ }^{17}$, l'absence de culture générale, les cloisonnements disciplinaires, tout cela a contribué à transformer les étudiantes et étudiants en sciences sociales en techniciens du social plutôt qu'en généralistes capables de trouver un sens aux diverses facettes du social.

Par ailleurs, il y a une dissociation de plus en plus importante entre l'enseignement et la recherche, ce qui ne contribue pas à assurer une relève intellectuelle ni à former intellectuellement les étudiantes et étudiants, principalement au premier cycle. En investissant de plus en plus dans la recherche puisque c'est ce qui est valorisé professionnellement, avec pour conséquence un désengagement sur le plan de l'enseignement, l'enseignement de premier cycle est de moins en moins dispensé par le corps professoral ${ }^{18}$. Ceci a pour effet de désintellectualiser la formation universitaire de premier cycle puisque l'accent est mis sur la transmission de connaissances plutôt que sur l'initiation au travail intellectuel.

16 Quoiqu'il porte sur les CEGEPs et non sur les universités l'article de Louise Lacour-Brossard souligne que, dans le secteur sciences humaines, on « retrouve une forte proportion d'étudiants qui rencontrent de sérieuses difficultés d'apprentissage et de réussite scolaires ». «Les étudiants en sciences humaines » Recherches sociographiques, XXVII, 3, 1986, p. 465.

17 Par simple cumul de crédits plutôt qu'en fonction d'un programme cohérent, la seule cohérence pouvant exister étant le fait de l'étudiante ou de l'étudiant.

18 Dans le réseau Université du Québec, les chargées et chargés de cours dispensent environ la moitié des cours. Dans d'autres universités, la situation est moins tranchée mais il y a tout de même un certain clivage entre l'enseignement et la recherche universitaires. 
En même temps, les sciences sociales, qui avaient joué un rôle déterminant dans la modernisation de l'État au cours de la révolution tranquille, ont subi les contrecoups de la perte du prestige étatique dans la société. Alors qu'avant la vague néo-libérale, le discours social dominant au Québec faisait de l'État le pivot du développement et de la modernisation, à partir du début des années 80 , ce rôle est conféré à l'entreprise privée, ce qui entraîne un glissement du discours du social vers la gestion. Ne s'agissant plus de comprendre mais de gérer, les sciences sociales sont devenues un discours de légitimation marginale, cédant la place aux gestionnaires dans la définition de l'avenir.

Cette marginalisation s'est accompagnée d'une technicisation. La recherche a de plus en plus été conçue sur le mode de l'application plutôt que de l'explication. Certes, pour certains la recherche reste encore trop fondamentale, mais les organismes subventionnaires font tout pour qu'elle devienne de plus en plus immédiatement utilisable et utilitaire. Dans ce sens, notre connaissance parcellisée du social et nos moyens d'investigation de la réalité sociale se sont beaucoup affinés au cours des dernières années, mais cette connaissance si elle sert, dans certains cas, à alimenter le pouvoir, contribue peu à introduire un débat social sur l'avenir collectif.

La conséquence d'une telle transformation de la fonction universitaire est que celle-ci a de plus en plus été vécue sur le mode de l'expertise, un phénomène qui a été concrétisé par le rôle particulier que la commission Bélanger-Campeau a conféré aux universitaires : à côté des acteurs sociaux exprimant des intérêts, les universitaires ont été appelés à fournir des avis autorisés à partir de leur « expertise ». Ce n'est pas la première fois que le phénomène se produit quoique, dans les commissions précédentes, les intellectuels en sciences sociales aient eu aussi leur mot à dire dans le rapport final, faisant partie des commissaires plutôt que d'en être réduits à un rôle de consultation externe au processus délibératif ${ }^{19}$.

L'expertise n'est pas un phénomène nouveau dans l'histoire récente des intellectuels québécois. La révolution tranquille a été aussi vécue sous le signe de la modernisation scientifique de l'État, les sciences sociales fournissant des modèles autorisés de la modernité. C'est au nom de la science et du progrès que les nouvelles élites ont remplacé les anciennes. Le savoir se muait en pouvoir et le mythe platonicien du philosophe sortant le monde de la caverne de la grande noirceur n'était pas bien loin.

19 A des fins de comparaisons, mentionnons que sur la commission Tremblay siégeait Esdras Minville et sur la commission Parent, Guy Rocher. 
Ce phénomène est très bien illustré dans l'analyse de Soulet qui compare les intellectuels québécois de la révolution tranquille aux Aufklärer et souligne leur rôle déterminant dans la modernisation de l'État

La mobilisation des classes moyennes, des techniciens aux experts, autour de l'État s'explique donc économiquement par la nécessité de constituer une infrastructure de production et socialement par les possibilités d'ascension collective qu'impliquait cette centration sur les savoirs et les compétences détenus par les classes moyennes ${ }^{20}$.

S'inspirant des analyses de Konràd et Szelenyi ${ }^{21}$, Soulet souligne la complémentarité de la construction étatique et de la consolidation des sciences sociales et le passage relativement aisé de la sphère intellectuelle à la sphère politique.

\section{Les pièges de l'idéologie}

$\underline{\text { Retour à la table des matières }}$

À l'opposé de cette expertise, la fonction intellectuelle a aussi été vécue sur le mode idéologique. Si certains intellectuels ont entrepris de «bâtir le Québec » moderne, pour reprendre le titre d'un rapport aujourd'hui oublié, d'autres ont entrepris de le critiquer au nom d'une autre conception de la modernité. Cette critique a pris essentiellement deux directions : le nationalisme et le socialisme, deux projets sociaux dans lesquels les intellectuels ont joué un rôle de premier plan et qui se sont tous deux heurtés à des échecs majeurs au début des années 80. Deux idéologies opposées également qui ont toutes deux prétendu au monopole explicatif et qui ont été le lieu à partir duquel les intellectuels ont été présents dans le social.

Aujourd'hui, entreprendre une critique de l'idéologie est certes plus facile que cela ne l'a été dans le passé. La pensée postmoderne ne nous a-t-elle pas habitués à la crise des récits explicatifs et à discréditer les idéologies au nom du microsocial. Mais il faut aller plus loin que ces lieux communs et voir le rôle que l'idéologie a permis aux intellectuels d'exercer. Cela permettra peut-être d'éviter de retomber dans le piège idéologique qui semble de nouveau hanter les intellectuels depuis l'échec de l'accord du Lac Meech.

Marc Henry Soulet, op. cit., p. 44.

21 Gyorgy Konràd et Yvan Szelenyi, La marche au pouvoir des intellectuels, Paris, Seuil, 1979. 
L'échec d'idéologies particulières peut d'abord se percevoir sur le mode événementiel. Au début des années 80, le nationalisme enregistre un premier échec de taille avec d'une part l'échec référendaire et d'autre part le rapatriement de la constitution canadienne sans l'accord du gouvernement québécois, ce qui consacre le succès de la vision centralisatrice du fédéralisme canadien développée par Trudeau. Par ailleurs, le mouvement syndical traverse péniblement la crise économique et tombe dans un corporatisme qui permet difficilement d'y voir un carrefour des forces de critique sociale. Quant à la gauche m.l., les organisations se dissolvent les unes après les autres, victimes de leur propre sectarisme et de la crise des systèmes politiques dont elles se réclamaient.

Toutefois, une telle approche événementielle ne suffit pas pour comprendre le silence des intellectuels dans les années 80 et le renouveau de la tentation idéologique dans les débats actuels. Ce qui est alarmant, ce n'est pas tant que les intellectuels se soient trompés (encore que l'erreur reste à prouver), mais qu'ils se soient trompés en prétendant détenir la vérité et en se considérant comme les porte-paroles de tendances sociales majoritaires.

Car il est indéniable que la question de la forme étatique la plus adéquate demeure encore pertinente. Certes, l'évidence de l'État-nation et son universalité ont été remises en cause, mais le modèle conserve son pouvoir d'attraction comme en témoignent les résurgences nationalitaires dans les pays d'Europe centrale et orientale. Même au Québec, ce mouvement que d'aucuns avaient cru pouvoir enterrer acquiert une nouvelle crédibilité politique. Le problème n'est pas tant que des intellectuels aient été ou soient encore nationalistes, c'est plutôt qu'ils abdiquent tout sens critique au profit de l'efficacité politique.

De même, ce n'est pas en fournissant un enterrement de première classe au marxisme et aux autres variantes de socialisme que l'on résoudra les inégalités économiques et sociales qui ne sont pas sans conséquences politiques. S'il paraît désormais plus que raisonnable d'entretenir des doutes sur la validité des solutions proposées par le marxisme, il n'en reste pas moins que les conditions sociales qui en ont favorisé l'émergence perdurent et qu'il importe de rechercher des modalités de justice sociale qui soient compatibles avec le maintien des libertés publiques et individuelles.

L'échec référendaire ou le rapatriement de la constitution canadienne peuvent être expliqués de façon rationnelle, là n'est pas la question. De même, l'on peut sociologiquement comprendre la perte d'influence sociale du mouvement syndical et la décomposition de la gauche m.l. Ce qui est moins justifiable, c'est le silence des intellectuels sur leur rôle idéologique dans ces deux cas. 
Le grand impensé demeure le rôle des intellectuels dans le développement d'une pensée socialiste et surtout du mouvement m.l. Sur le plan politique, l'effet du «marxisme-léninisme » a été une véritable catastrophe. D'une part, cela a fortement discrédité toute pensée de transformation sociale radicale et a justifié l'engouement béat pour un libéralisme qui connaît aussi sa part d'échec social ${ }^{22}$. D'autre part, cela a fortement affaibli des organisations qui poursuivaient des objectifs restreints mais produisant des résultats probants dans la réduction des inégalités.

Il est devenu maintenant courant de critiquer le marxisme comme idéologie en s'autorisant de la décomposition des régimes s'en réclamant pour ce faire. Cela ne nous mène pas bien loin, d'autant que l'on peut déceler autant d'échecs sociaux dans le camp capitaliste. Il m'apparait beaucoup plus utile d'aborder la question par le biais de l'effet politique de la «marxisation » des intellectuels québécois.

Un premier effet, c'est la persistance de la foi du charbonnier. Le monde intellectuel québécois est passé en moins de vingt ans de l'orthodoxie catholique à un autre type d'orthodoxie ; cela a certes une fonction de réassurance : le passé, le présent et l'avenir ont un sens et peuvent acquérir une intelligibilité. Mais le revers de la médaille, c'est l'absence de curiosité. Le monde est fini, clos; l'imprévisible et l'événement n'y ont pas leur place. On ne cherche plus, par le biais du débat, à concourir à la formation de l'opinion publique. On cherche à imposer. La certitude de la vérité entraine l'économie de sa recherche. Le politique se réduit donc aux techniques permettant de faire triompher sa vérité.

Hannah Arendt, dans son analyse du totalitarisme, montrait le rôle de l'idéologie dans la consolidation du totalitarisme en soulignant d'abord le risque que l'idéologie fait courir à la pensée dont elle représente en quelque sorte la négation

Le danger d'échanger la nécessaire insécurité où se tient la pensée philosophique pour l'explication totale que propose une idéologie et sa Weltanschauung, n'est pas tant le risque de se laisser prendre à quelque postulat généralement vulgaire et toujours précritique, c'est celui d'échanger la liberté inhérente à la faculté humaine de penser pour la camisole de la logique, avec laquelle l'homme peut se contraindre lui-même presque aussi violemment qu'il est contraint par une force extérieure à lui ${ }^{23}$.

22 On n'a qu'à penser aux taux de chômage qui prévalent dans la quasi-totalité des pays industrialisés de même qu'au nombre important d'Américains privés de tout accès à des soins médicaux faute d'argent. Et encore, j'ai la générosité de ne puiser mes exemples que dans les pays les plus développés.

23 Hannah Arendt, Les origines du totalitarisme, Paris, Seuil, 1972, p. 218. 
C'est ce qui l'amènera à soutenir que « [le] sujet idéal du règne totalitaire n'est ni le nazi convaincu, ni le communiste convaincu, mais l'homme pour qui la distinction entre fait et fiction et la distinction entre vrai et faux (...) n'existe plus $24 »$.

Certes, cette menace totalitaire était plutôt circonscrite au Québec, mais il est significatif que ce soit les intellectuels qui en aient le plus été affectés. Il est particulièrement dangereux, pour quelque société que ce soit, que ceux et celles qui devraient faire profession de penser adhèrent à la non-pensée.

Cela a pour conséquence d'engendrer une baisse de la participation politique. Malgré toutes les critiques qu'on peut lui adresser, la révolution tranquille a tout de même eu pour effet que la population s'est un moment crue artisane de son destin et a commencé à s'organiser pour améliorer sa situation. En essayant de noyauter tout ce qui bougeait socialement et d'instrumentaliser l'ensemble des luttes sociales, le mouvement m.l. a contribué à une démobilisation politique remarquable. Plutôt que de se faire manipuler, plusieurs ont préféré rentrer chez eux et se replier sur leur vie privée, devenant sceptiques face à toute tentative de changement social.

Il serait intéressant de se demander si une composante de la crise des idéologies ne provient pas de l'incapacité des intellectuels à admettre qu'il s'étaient trompés. En tout cas, il est évident que l'absence de débat, puisque le propre de la parole autorisée est de circuler à sens unique, ne favorisait pas la réflexion sur les pratiques antérieures et laissait peu de place à une attitude autre que la démission.

Quant à la pensée nationaliste, elle a eu à pâtir du désistement des intellectuels. La question nationale s'est progressivement vidée de son contenu social et culturel pour devenir un discours d'efficacité gestionnaire qui voit dans le lien fédéral canadien une entrave au développement économique du Québec. Le nationalisme a de plus en plus une texture de froide instrumentalité.

24 Ibid. p. 224. 


\section{Des voies d'intervention}

$\underline{\text { Retour à la table des matières }}$

Dans les deux cas de figure, celle de l'expertise et celle de l'idéologie, l'intervention des intellectuels en politique s'est avérée désastreuse parce qu'un élément essentiel a été perdu de vue. La scène politique n'est pas le lieu de la vérité, mais celui du débat ; elle ne devrait pas être celui de l'unicité mais celui de la pluralité. Il est donc problématique de s'autoriser de sa fonction sociale pour prétendre avoir une opinion plus valable que celle de son voisin, à tout le moins si l'on entrevoit la politique comme un processus démocratique fondé sur l'égalité des participantes et participants au débat.

Ces considérations sur les pièges de l'idéologie et de l'expertise ne tendent cependant nullement à préconiser le silence. Il faut toutefois distinguer entre les ordres de discours. Les discours de l'idéologie et de l'expertise se réclament tous deux de la vérité et il importe peu ici que cette vérité relève dans un cas de l'« Histoire » et dans l'autre de la «science». Ce que je qualifierais de fonction critique, c'est la capacité de susciter des débats publics, « de rendre compte des valeurs sous-jacentes à son travail, et de leur relation avec les valeurs de la société » ${ }^{25}$. Or c'est ce débat public qui fait cruellement défaut dans le contexte politique actuel, à moins de penser que la question constitutionnelle se limite à une question de partage de juridiction, de partage du déficit ou de gestion de la monnaie.

Ce qui est révélateur dans les démarches proposées par la Commission Bélanger-Campeau, c'est l'exclusion du débat public, la seule place concédée à la population étant de se prononcer lors d'un référendum dont l'enjeu paraît de plus en plus nébuleux. Or c'est ce débat public que nous devons contribuer à faire émerger, peu importe la position constitutionnelle que l'on adopte ${ }^{26}$. Dans une situation politique caractérisée par une crise de légitimité, nous devons faire en sorte que les valeurs démocratiques l'emportent à la fois pour des raisons civiques,

Tzvetan Todorov, Les morales de l'histoire, Paris, Grasset, 1991, p. 279.

À cet égard, le rapport Beaudoin-Edwards n'est pas plus de nature à susciter le débat public que celui de la commission Bélanger-Campeau. Ces deux rapports préfèrent s'en remettre aux seuls gouvernements. 
dans la mesure où la démocratie est le seul régime politique qui autorise une discussion sur ses fondements, et pour des raisons plus étroitement professionnelles, puisque la liberté de penser, si nécessaire à notre travail, est mieux protégée dans ce type de régime que dans n'importe quel autre.

On pourrait certes rétorquer que personne ne remet en cause la démocratie dans le débat constitutionnel actuel. Sur le plan formel, c'est exact. Sur le plan pratique, la situation est un peu plus complexe: le processus de modification constitutionnelle relève de politiciens dont la légitimité est elle-même remise en cause et surtout, il semble y avoir consensus pour faire silence sur le contenu de tout projet politique.

C'est ce silence qu'il faut dissiper si nous voulons contribuer à susciter un débat public. Il faut parvenir également à faire en sorte que les questions soulevées ne tournent plus autour de la notion d'identité, mais plutôt sur les modalités du vivre ensemble de la collectivité. Bref, il faut que le débat constitutionnel parvienne à s'arrimer aux enjeux sociaux concrets auxquels la société québécoise se trouve confrontée.

On peut nommer ici certains de ces enjeux, mais la liste ne saurait être exhaustive : l'attitude face à l'immigration, le rapport entre «blancs » et « autochtones », la place des femmes dans les structures politiques et sociales, le rôle des régions, le rôle et la responsabilité des pouvoirs locaux, de nouveaux modes de représentation politique. Je me limite même volontairement aux questions qui doivent assez immédiatement connaître une concrétisation institutionnelle laissant de côté les questions reliées à l'environnement et au développement économique.

Face à ces questions, notre première tâche est non pas d'apporter des réponses, mais de susciter un débat. Il n'y a aucune solution simple ni générale à ces questions. Elles ne peuvent être réglées ni par une politique spécifique ni une fois pour toutes. Cependant, elle doivent demeurer présentes dans l'élaboration des politiques et des mesures sociales. C'est pourquoi nous devons veiller à ce qu'elles continuent (ou commencent) à être des interrogations qui traversent le corps social. Elles ne doivent surtout pas être reléguées aux lendemains du débat constitutionnel mais contribuer à lui donner un peu de substance. Il s'agit pour nous de se référer «aux principes constitutifs de la société présente, en l'occurrence aux principes démocratiques, pour critiquer leur réalisation imparfaite dans la vie de tous les jours ${ }^{27}$ ».

27 Tzvetan Todorov, op. cit., p. 289. 
Jouer un rôle intellectuel, surtout dans les sociétés modernes qui se sont constituées dans le rejet de toute transcendance, c'est amener la société à s'interroger sur elle-même, ce qui constitue le moyen pour elle d'être vivante, tout en devant reposer sur des institutions stables.

Ce rôle est certes moins glorieux que de prophétiser l'avenir ou de façonner le présent en exerçant des responsabilités politiques. N'aspirer ni au rôle du prince ni se complaire dans ceux de conseiller du prince ou de prophète représente peut-être une nouveauté pour les intellectuels québécois, mais cela ne constitue-t-il pas la condition nécessaire au maintien d'une fonction intellectuelle dans la société ?

Nous avons une responsabilité sociale, comme membres d'une collectivité humaine et à ce seul titre. Notre action ne doit pas viser à assener des vérités mais plutôt à disséminer dans le corps social la faculté de penser. Cela devrait devenir notre «déformation professionnelle». C'est principalement à ce titre que la société pourra juger de notre action et nous demander de rendre des comptes.

Fin du texte 